\title{
A function-first look at tumor heterogeneity
}

Fate maps of human brain cancer cells, transplanted in mouse, challenge traditional views of tumor genomic heterogeneity.

Glioblastoma is a recalcitrant, aggressive cancer of the brain. Despite intense research, little headway has been made into its treatment. Much of the blame for this has fallen on the nature of the disease, which is synonymous with diversity; the very "idea of glioblastoma, the old name 'multiforme', being one of the classic tumors with tremendous cellular heterogeneity," observes Peter Dirks, a cancer researcher at the Hospital for Sick Children in Toronto.

For Dirks and his collaborator Benjamin Simons at the University of Cambridge, a critical question is what gives rise to this heterogeneity. The researchers adopted a barcoding strategy to trace cell clones during tumorigenesis in vivo, and they focused on measuring the functional manifestations of cancer-self-renewal, invasiveness and response to therapy-in addition to assessing genomic differences.

Glioblastoma tumor growth is thought to depend on a stem cell population that sits atop a proliferative hierarchy. The Dirks laboratory helped to establish the existence of these stem cells, which have higher tumorinitiating potential and greater resistance to therapeutics than other cells in the tumor. To characterize the hierarchy, they and other groups relied heavily on marker-based cell sorting, which is technically demanding, or on using mouse models. "We needed new methods...new ways of looking at the human cells," says Dirks.

To follow human clones in vivo, the researchers infected primary glioblastoma cells with lentiviral vectors bearing random sequences, such that each cell was labeled with a unique barcode. The cells were then immediately injected into one brain hemisphere of an immunodeficient mouse and allowed to form tumors before being collected for sequencing. Spike-in controls were used to estimate the size of clones from barcode readout counts, and the process was repeated for two serial passages. Critical guidance with the viral barcoding came from Connie Eaves and Martin Hirst at the University of British Columbia, who pioneered the technique in solid tumors.

The approach was ambitious; it took over four years, and the serial transplantations alone represented a year of work with many risks. "Maybe it was rather naïve, but we didn't know what it was going to show until we finally put all the sequencing together," says Dirks. What they found was striking-there was enormous heterogeneity in clone sizes from all six patient tumors, but the distributions could be fit with a simple equation and a single adjustable parameter. "That's quite a deep observation," says Simons. "What it suggests is that although cells may be behaving in a stochastic manner...they're obeying some defined set of rules."

The results refute the model that glioblastoma subclones bearing different mutations evolve based on differential fitness in the brain. Instead, the results suggest that a single stem cell subpopulation drives a tumor's heterogeneity through neutral evolution. The form of the clone size distribution, a negative binomial, is consistent with a hierarchy composed of slowly self-renewing stem cells that generate a rapidly dividing intermediate population that in turn produces short-lived progeny. The potential for the tumors to invade the neighboring hemisphere also appeared to coincide with this intrinsic self-renewal capacity.

The researchers tested serial transplants in the presence of temozolomide, a first-line glioblastoma treatment. Most clones were sensitive, but unlike what is seen in mouse models, the clones maintained their relative size distribution, which indicated that the drug had little effect on the hierarchy. However, about $5 \%$ of clones fell outside the distribution both before and after treatment,

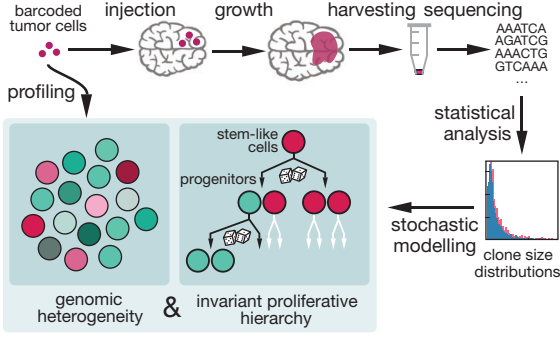

Clonal analysis helps to characterize glioblastoma and find treatments. Adapted from Lan et al. Springer Nature (2017).

and these could represent populations that expand through chemotherapy.

These observations inspired lead author Xiaoyang Lan, a PhD student in the Dirks lab, to generate primary glioblastoma stem cell cultures based on this difference in behavior, rather than on individual mutations, and to screen for drug sensitivity. Chromatin accessibility data pointed to shared epigenetic differences within cultures; indeed, the team found two epigenetic modifiers, one affecting each clonal type, which together were effective in preventing the growth of both types.

"So much emphasis is placed on understanding the mutational heterogeneity of tumors," says Simons. But their work, though limited to a few glioblastoma types and patient tumors, demonstrates the importance of considering cell function. Dirks agrees. "Everyone's steeped in 'more sequencing will find more mutations that will identify small molecules..." he says, while few targeted therapies have been nominated. The clonal analysis gives Dirks hope of finding a common program that is "agnostic to mutation, or that mutations all funnel through the same state...If we can understand then that state, the driving state, we can actually think of stopping it."

Tal Nawy

RESEARCH PAPERS

Lan, X. et al. Fate mapping of human glioblastoma reveals an invariant stem cell hierarchy. Nature 549, 227-232 (2017). 